\title{
Experimental characterization towards an in-fibre integrated silicon slab based all-optical modulator
}

\author{
H. Pinhas, Y. Danan, M. Sinvani, M. Danino and Z. Zalevsky ${ }^{*}$
}

\begin{abstract}
Background: Due to the industrially increasing interest in optics communication the field of all-optical modulation is becoming more and more popular. However, no in-fibre integrated miniaturized devices which provide current solutions were suggested as all-optical modulators with sufficient modulation depth and with low power consumption.

Methods: In this paper, an all-optical silicon modulator with improved modulation depth is presented. The modulator will be developed as an in-fibre device for optics communication applications. The modulator is a silicon slab, 50 and $470 \mu \mathrm{m}$ thick which is coated on both sides to get an improved Fabry-Perot resonator for laser beam at wavelength of $1550 \mathrm{~nm}$. The modulator operation is based on the plasma dispersion effect which is induced by a pulsed visible laser beam acting as the pump for the creation of free charge carriers.

Results: We have proved the coating withstands high energy fluency of the laser pulse. The different recombination and heat processes are examined. Our silicon based Fabry-Perot resonator increases the intrinsic c-Si finesse to 30. The improved finesse is shown to have significant effect on the modulation depth up to $12 \mathrm{~dB}$.

Conclusions: To the best of our knowledge this is the first experimental feasibility study of an all-optical modulator with sufficient modulation depth and with low power consumption which is also an in-fibre integrated miniaturized device based upon Si slab. Extensive experimental and numerical studies presented in this paper examine the influence of thermal effects and the sample parameters on the response time and on the modulation depth and the power consumption of the in-fibre integrated device. Apart from being a modulator, the proposed device can also act as tunable spectral filter.
\end{abstract}

Keywords: Optical system design, Interference, Modulation, Transmission, Attenuation

\section{Background}

Today, most optical modulations are achieved through the use of electrical circuits controlling the voltage applied on the modulator [1,2]. Nevertheless, due to the industrially increasing interest in optics communication the field of all-optical modulation is becoming more and more popular [3-7]. The most important restriction in designing the all-optical modulator is the desire to realize it on Silicon $(\mathrm{Si})$ in order to allow its integration with microelectronic circuitry.

One way to implement the optical modulator is by ring resonators in Si that use the plasma dispersion effect.

\footnotetext{
* Correspondence: zeev.zalevsky@biu.ac.il

Faculty of Engineering and the Nano-Technology Center, Bar-llan University, Ramat Gan 52900, Israel
}

Lipson et al. [8-10] have shown optical modulation in $\mathrm{Si}$ ring resonators in which light is confined and resonates in order to enhance the optical modulation effects. They used Silicon-On-Insulator (SOI) ring resonator and in order to get the optical effect they shone with pump power of $25 \mathrm{pJ}$. Schönenberger et al. [5] demonstrated SOIs devices which have reached power requirements of $6 \mathrm{fJ}$ for $3 \mathrm{~dB}$ modulation depth with a switch-on time of few picoseconds and recovery time limited by the free charge carriers (FCC) life time being 500 ps. It should be noted that similar works were also done with different materials such as the CMOS compatible amorphous silicon [11-13]. Even though the absorption in a-Si: $\mathrm{H}$ is an order of magnitude above that of c-Si, $1.63 \cdot 10^{-16} \mathrm{~cm}^{2}$ compared to $1.45 \cdot 10^{-17} \mathrm{~cm}^{2}$, they mainly use the electro-absorption (EA) effect and not the 
electro-refractive (ER) effect which has a more dominant influence to the modulation [9], which reduces the efficiency of the device.

Other than ring resonators, Fabry-Perot (FP) resonators can act as optical modulators as well; Gibbs et al. have shown all-optical FP modulation in GaAs [14]. Si based FP with modulation depth of almost $3 \mathrm{~dB}$ with $400 \mu \mathrm{J}$ pump energy was demonstrated later on by Aharoni et al. [15]; In order to improve the modulation efficiency, mirrors were added directly on both sides of the Si slab. Adding mirrors indeed increased the signals attenuation but induced critical alignment difficulties.

These various methods represent great progress in the all-optical modulation field. However, some of the SOI based modulators are governed by semi-conductors planar complex structures. These structures contain metals and insulators as part of 3D structures with features in a nanometer scale arranged tightly on a chip which makes them expensive and hard to produce [15]. Others, simpler SOI based modulators, $[3,6,8]$, cannot be fabricated as in-fibre modulators since they have to be implemented as part of an SOI chip. The simpler FP modulators which is Ge based, could be integrated on top of a fibre exit, lost the advantage of $\mathrm{Si}$ being the essential material in integrated chips. The main problem with Si based FP modulators is that they are not efficient enough to function as modulators in fibre systems such as fibre lasers or communication systems.

In this paper, to the best of our knowledge, we demonstrate for the first time the experimental feasibility of a simplified coated Si slab all optical modulator with sufficient modulation depth for compact, low power consumption integrated in-fibre optics communication system. Throughout the paper, discussion of the new difficulties that arise in the case of fibre based Si slab modulator will be addressed. The advantage of a slab resonator, rather than a ring resonator, lies in the simplicity of the device which, for instance, can be deposited onto a fibre avoiding the SOI substrate and coupling of tapered waveguides (resulting in high transmission losses). Different silicon slabs with various thicknesses and finesses are examined and their modulation processes are explained with consideration to the different recombination mechanisms [16]. Another advantage is that the device is substrate free and together with its simplicity it will have low manufacturing costs. Moreover, the Si slab is coated in order to improve the $\mathrm{Q}$ factor (the finesse) of the device and lower the modulation power requirements for the in-fibre systems. We also present extensive experimental and numerical study that examines the influence of thermal effects and type of coating on the response time and on the modulation depth and the power consumption of the device. Apart from being a modulator, the proposed device can also act as tunable spectral filter.

\section{Methods}

Since Si has no electro-optical, modulation is achieved by changing the complex refractive index through a temporary increase in the concentration of FCC which is obtained by applying laser pulse as a pump. This effect is known as the plasma dispersion effect (PDE) [17-19]. The PDE is weak in Si and requires significant enhancement for practical modulation which in our case is enhanced via the FP resonator.

There are two main effects derived from the PDE. The first is the ER effect and the second is the EA effect. The latter occurs due to the change in the imaginary part of the refractive index, $i \alpha \lambda / 4 \pi$, which modifies the absorption coefficient through free carrier absorption $(\Delta \alpha)$ while the former occurs due to change in the real part of the refractive index through free carrier change of index $(\Delta n)$ of the $\mathrm{Si}$. Where $\Delta n$, and $\Delta \alpha$ are derived from the well-known Drude-Lorentz equation [20] and were determined empirically as [19]:

$$
\begin{gathered}
\Delta n=-8.8 \times 10^{-22} \cdot \Delta N_{e}-8.5 \times 10^{-18} \cdot\left(\Delta N_{h}\right)^{0.8}, \\
\Delta \alpha=8.5 \times 10^{-18} \cdot \Delta N_{e}+6 \times 10^{-18} \cdot \Delta N_{h} .
\end{gathered}
$$

where $N_{e}, N_{h}$ are the electron and hole concentrations, respectively and $\lambda$ is the optical wavelength of the information signal, the probe (this wavelength differs from the wavelength of the pump beam).

The transmission of a FP interferometer (together with the Beer-Lambert law - to incorporate absorption in the system) can be expressed as follows:

$$
\frac{I_{\text {out }}}{I_{\text {in }}}=\frac{\left(1-R_{1}\right)\left(1-R_{2}\right) e^{-\alpha d}}{\left(1-\sqrt{R_{1}} \sqrt{R_{2}} e^{-\alpha d}\right)^{2}+4 \sqrt{R_{1}} \sqrt{R_{2}} e^{-\alpha d} \sin ^{2}\left(\frac{\delta}{2}\right)}
$$

Where $R_{1}, R_{2}$ are the reflectivity of FP planes from both sides of the slab, $\alpha$ is the $\mathrm{Si}$ absorption coefficient, $\mathrm{d}$ is the diffusion depth of the FCC created by the pump laser pulses and $\delta$ is the round trip phase (of the probe signal) which equals to $\delta=2 k L n$ (where $k=2 \pi / \lambda$ and $\mathrm{L}$ is the thickness of the Si slab). A modification in Eq. (2) due to the PDE will be Si refractive index $\mathrm{n}=\mathrm{n}_{0}+\Delta \mathrm{n} \cdot \mathrm{d} / \mathrm{L}$ and Si absorption coefficient $\alpha=\alpha_{0}+\Delta \alpha \sim \Delta \alpha\left(\alpha_{0}\right.$ is negligible at $1550 \mathrm{~nm}$ which is the wavelength of the probe).

As increase of charge is always associated with power dissipation, a phase modulator based only on PDE is problematic because the thermo-optic effect (TOE) will always be present. In the TOE the refractive index is varied by applying heat to the material. The problem is that the TOE refractive index change is slower (microseconds) than that of the PDE (nanoseconds). However, it can still be used to cause modulation or at least to counter act the ER effect when necessary [21, 22]. 


\section{Results}

The following presented experimental setups are divided into two setups: free space modulation, shown in Fig. 1 and in fibre modulation, shown in Fig. 2.

Both of the setups use the same probe, pump and coated Si slab. As a probe we used $5 \mathrm{~mW} C \mathrm{CW}$ laser beam at $1550 \mathrm{~nm}$ ("HP $8168 \mathrm{~F}$ " tunable laser) with $2 \mathrm{~mm}$ diameter. The detector is "PDA400" with $35 \mathrm{~ns}$ rise time. As the pump we used laser pulsed illumination, coming from Nd-YAG laser (Surelite Continuum I-20) which illuminates the Si slab at the same point as the incident point of the IR probe beam as illustrated in Fig. 1a. As can be seen in Fig. 1b the IR light beam exiting a collimator and passing through a lens which focuses the light beam onto the slab. The pump light beam alignment onto the same spot as the IR light beam is done with two mirrors. The pump emits $0.3 \mathrm{~mJ}$ energy, $532 \mathrm{~nm}$ wavelength, 5-7 ns long coherent light pulses and its light beam diameter is $5 \mathrm{~mm}$.

Once the all-optical modulation is achieved in free space we advanced to fibre modulation. The in-fibre integrated device prototype can be seen in Fig. 2a. The pump and the probe light beams are coupled with a Y coupler. As seen in Fig. 2b, from the right, a CW IR light beam (solid red) is transmitted in a fibre and coupled with the pump light beam (green). The pump modulates the IR beam through the Si slab and the modulated IR light beam exiting the slab to the left (dashed red).

The power damage threshold of the coated slab is about $0.5 \mathrm{~J} / \mathrm{cm}^{2}$ for the pump 5-7 ns long pulses. It was not obvious that this coating can withstand such intensities while all the energy is absorbed in the skin layer of the coated Silicon. The coatings are made of 18 dielectric layers of

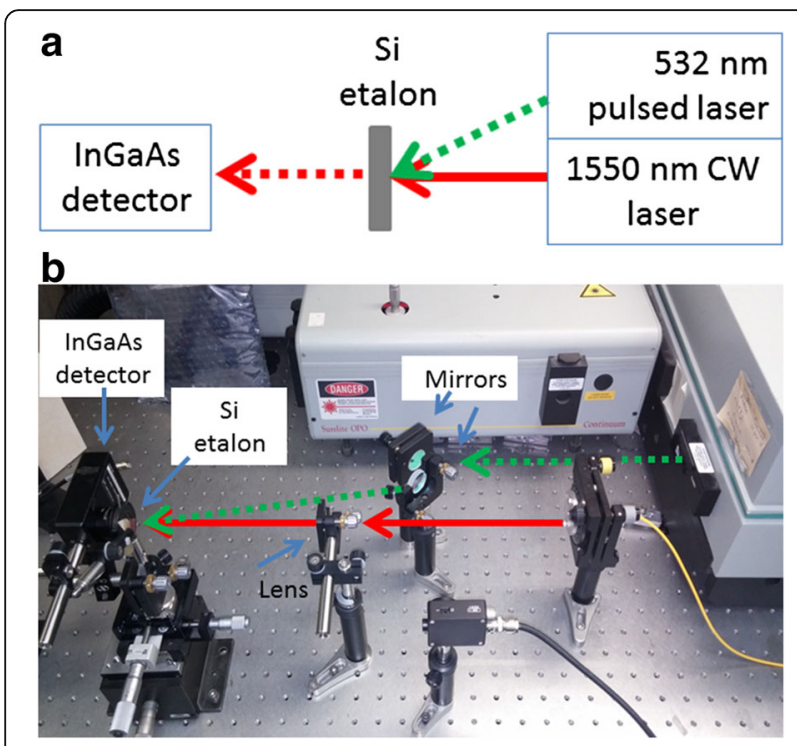

Fig. 1 Free space modulation setup: a Scheme of the experimental setup. b The experimental setup

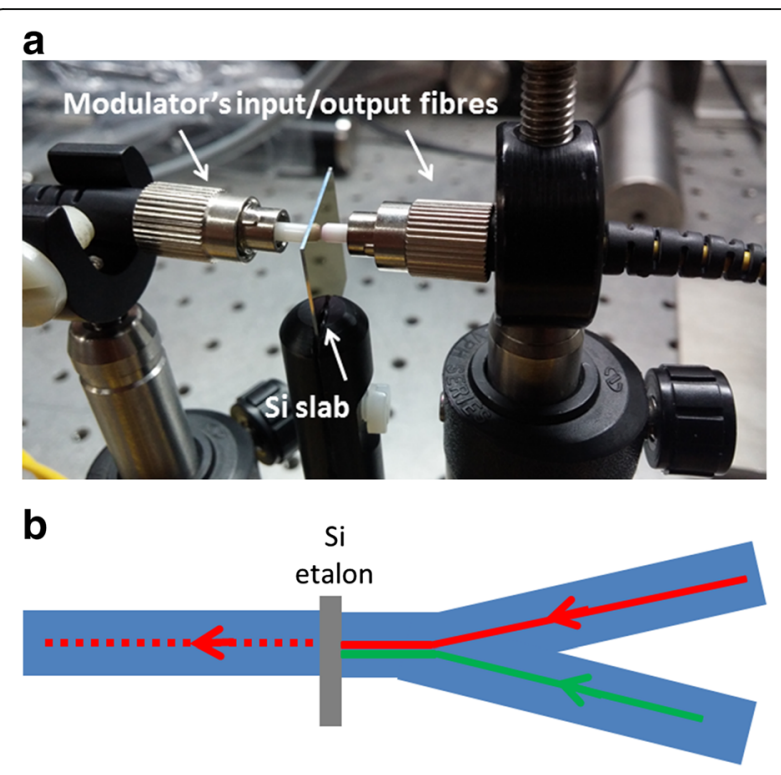

Fig. 2 Fibre modulation setup: a the experimental setup composed of a Si slab integrated into an input and output fibres. $\mathbf{b}$ Scheme of the probe and pump beams propagation in the device

$\mathrm{Ta}_{2} \mathrm{O}_{5}$ and $\mathrm{SiO}_{2}$ with a total material thickness of $1710 \mathrm{~nm}$ and $2250 \mathrm{~nm}$, respectively, on each side of the Si slab.

\section{Simulations}

In order to simulate the pump influence on the IR transmission the experiment described above was simulated. The simulation of the probe transmission through the $\mathrm{Si}$ slab versus wavelength was done using MATLAB (R2013b). In this simulation, Si penetration depth of around $1 \mu \mathrm{m}$ at wavelength of $532 \mathrm{~nm}$, FCC diffusion depth of $10 \mu \mathrm{m}$ at $10 \mathrm{~ns}$ pulse width and quantum efficiency of 0.8 to extract the FCCs, were used. The FCCs concentration gives us the change in absorption and in the real part of the refractive index (Eqs. (1) and (2)). In Fig. $3 \mathrm{a}$ and $\mathrm{b}$ we present the all-optical modulation simulation results for two different thicknesses of $\mathrm{Si}$ slabs. As seen in these figures, the free spectral ranges (FSR) of the $50 \mu \mathrm{m}$ thick sample is $6.9 \mathrm{~nm}$ and for the $470 \mu \mathrm{m}$ thick sample $0.73 \mathrm{~nm}$ and their shifts from the resonance wavelength are about $-2 \mathrm{~nm}$ and $-0.2 \mathrm{~nm}$, respectively. Since these two slabs have the same finesse as part of the parameters defining the cavities, they have the same ratio of full width half maximum (FWHM) as the FSR ratio.

The FSR is inversely proportional to the thickness of the resonator, and thus the FWHM in a nominal finesse. In addition, any change in refractive index translates into a wavelength shift from the cavity resonance according to Eq. (2). Thus, transmission in cavities with wider FWHM will have weaker dependence on refractive index changes. This is seen in the literature when higher 

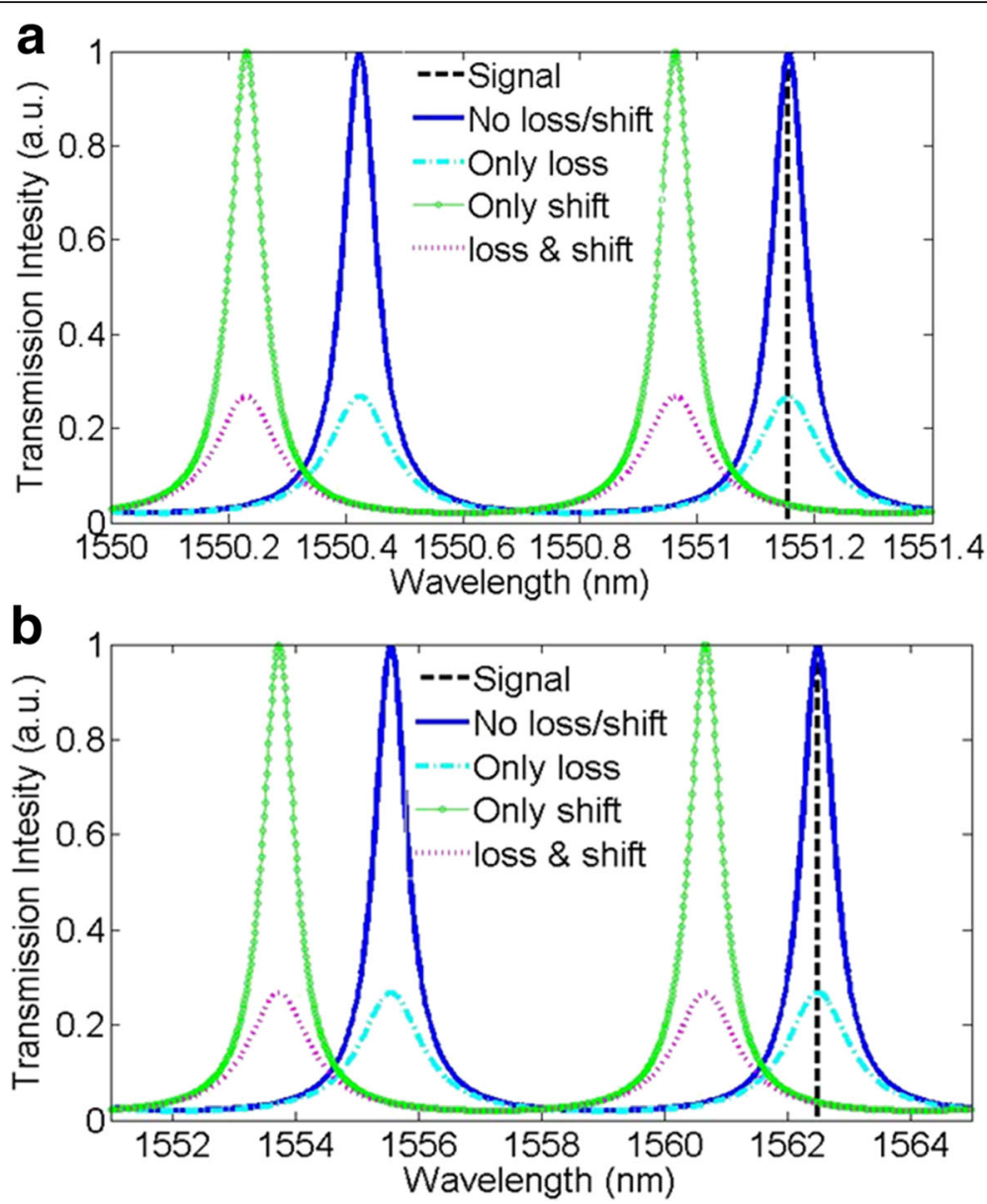

Fig. 3 Matlab simulation: a Transmission intensity vs. wavelength of a coated slab, $470 \mu \mathrm{m}$ thickness and $\mathbf{b} 50 \mu \mathrm{m}$, Finesse $\simeq 10$

temperature is needed to induce same phase shift in thinner slabs, through the TOE. Thus, any change in the refractive index due to TOE and PDE is supposed to have weaker effect in thinner slabs. However, our simulation results (see Fig. 3) show that the relative change in phase in slabs with different thicknesses is the same.

This can be explained as follows: Even though the diffusion depth of FCCs is the same, the relative part of the changed refractive index compared to the slab thickness is different i.e. the relative part of the diffusion depth of FCCs in the $50 \mu \mathrm{m}$ thick slab is $1 / 5$ and in the $470 \mu \mathrm{m}$ thick slab is $1 / 47$.

A scheme of the simulation of the heat dissipation across the slab using COMSOL program is presented in Fig. 4a. It shows how heat distributes on the surface and along the probe light beam. Averaged temperature across the entire probe light beam path inside the slab vs. time is shown in Fig. 4b. The change in refractive index due to heat concentrated in one spot along the beam path is equivalent to the change due to heat distributed along the entire beam path. Thus, we calculated the overall temperature change along this path. The heat induced by the pump decreases to less than $0.1^{\circ}$ after 100 ns. This temperature translates to about $2 \times 10^{-5}$ increase in refractive index which yields a negligible modulation depth of only $0.06 \%$. Hence, modulation rates up to $5 \mathrm{MHz}$ can be achieved without significant heat effects. Notwithstanding, with such rates heat might accumulate and increase the temperature, however, the amount of heat exiting the system will increase as well so the system will return to its thermal equilibrium.

\section{Experimental results}

Transmission through a Si slab is shown in Fig. 5, demonstrating the resonance effect. The slab, acting as an etalon resonator, more than doubles the effective optical path that the probe light travels inside the slab, increasing the resonator sensitivity to optical effects.

Nonetheless, in the case of a bare Si slab (Fig. 5a) this factor is not sufficient for substantial modulation. Transmission through a coated $\mathrm{Si}$ slab results in improved finesse as can be seen in Fig. 5b. The peak separation is 

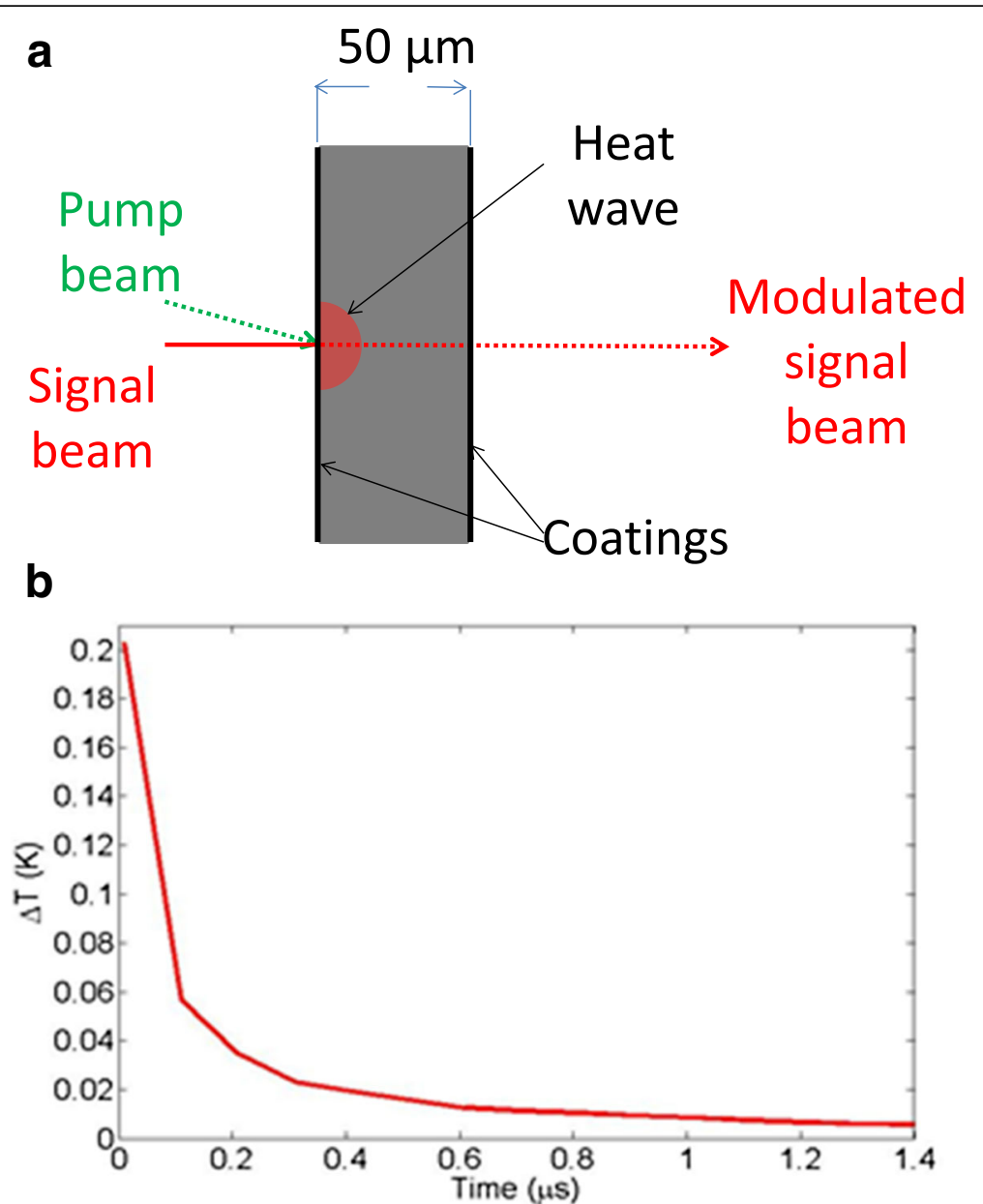

Fig. 4 COMSOL simulation: a Heat wave on the slab due to pulse beam and $\mathbf{b}$. Averaged temperature across the entire light beam path inside the slab vs. time

still $0.73 \mathrm{~nm}$ because the etalon thickness remained the same. However, the FWHM has decreased to $0.07 \mathrm{~nm}$ due to the coating, giving a finesse of 10 which is 4 times than that of the one of the uncoated slab.

The experimental measurement of the transmission of the probe laser beam through the sample as a function of time can be seen in Fig. 6. The probe wavelength is tuned to give the maximum transmission according to the resonance spectrum [23]. At time $t=0$, the pump pulse illuminates the Si slab and FCCs are generated and thus the pump attenuates the probe transmission. The attenuation speed is determined by the detector rise time of 35 ns. An additional process is driven by the TOE; once the fast surface recombination ends, the heat takes a more dominant role.

The modulation depth in this $470 \mu \mathrm{m}$ thick slab is about $12 \mathrm{~dB}$ with transmission intensity to full recovery is beyond $1 \mu \mathrm{s}$, that is, beyond the scale of this figure. This recombination process is slow and hence exhibits a disadvantage because of its modulation rate limitation.
Hereafter, a faster recombination process is shown in a thinner slab $(50 \mu \mathrm{m})$. In this experiment the modulation is done on two $50 \mu \mathrm{m} \mathrm{Si} \mathrm{slabs} \mathrm{with} \mathrm{different} \mathrm{finesses.}$ The experimental results are shown in Fig. 7. The probe wavelength is tuned to give maximum transmission (resonance) and the pump pulse illuminates the slab at $t=0$ which results in attenuation in transmission of $12 \mathrm{~dB}$ and a full recovery after about $600 \mathrm{~ns}$. The recovery time in these slabs is faster than in the case of the thicker slab even though in the simulation section the same relative shift in the resonance spectrum between the $50 \mu \mathrm{m}$ and $470 \mu \mathrm{m}$ slabs was seen (Fig. 3). A similar behavior was seen in the work of Aharoni et al. [12]. They explained this difference by a more dominant surface recombination in the thinner slabs. That is, the change in recombination rates from fast to slow is due to changes in the recombination processes, from surface to bulk recombination [24].

The finesse has an important role in determining the modulation characteristics such as pump pulse intensity, 


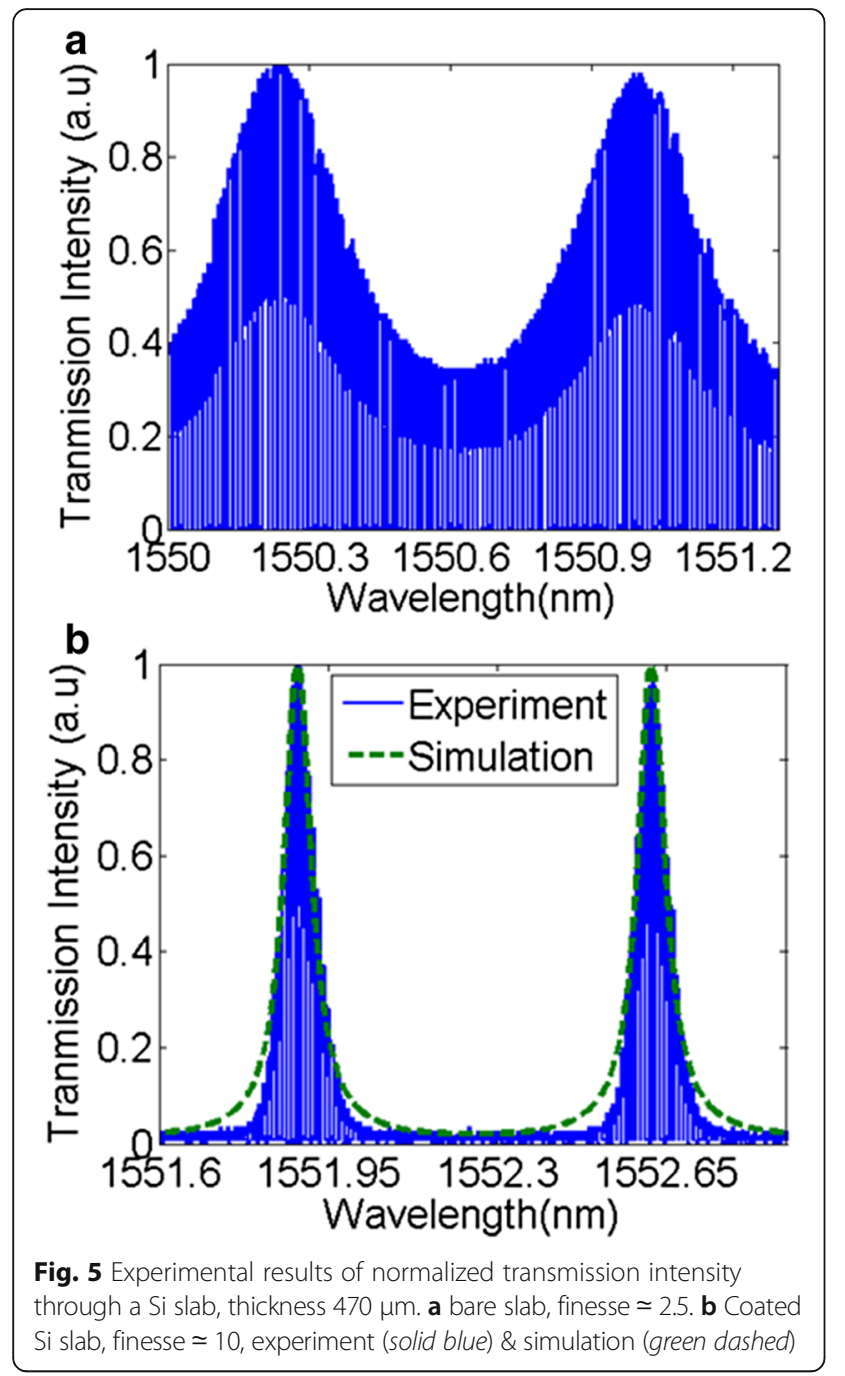

wavelength shift and recovery time. In order to better understand how these parameters affect the modulation and the recovery time, experimental results in two $\mathrm{Si}$ slabs with different finesse were conducted. These experimental results can be seen in Fig. 8.

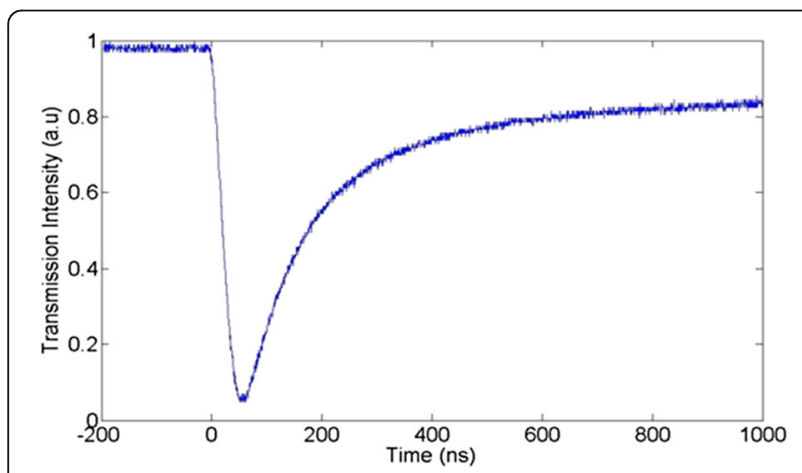

Fig. 6 Attenuation due to optical pump induced plasma dispersion yielding $12 \mathrm{~dB}$ loss in transmitted intensity



Fig. 7 Probe intensity attenuation due to optical dispersion in $50 \mu \mathrm{m}$ thickness Si resonator with finesse of: $\mathbf{a} 10$ and $\mathbf{b} 30$

Because of the same etalon thickness, the slabs with the different finesse have the same FSR $(6.9 \mathrm{~nm})$, however, they have different FWHM with the inverse ratio of their finesse: finesse of $7 \& 30$ with FWHM of $1 \mathrm{~nm} \&$ $0.23 \mathrm{~nm}$, respectively. When the finesse is increased from 7 to 30, the modulation depth is expected to increase as well since at a nominal pump power the same change in $\Delta \lambda$ should result in a greater decrease in intensity.

The wavelength shift from resonance wavelength to less than $5 \%$ of transmission in the etalon is $\Delta \lambda=-$ $1.7 \mathrm{~nm}$ in the etalon with finesse of 7 and $\Delta \lambda=-0.7 \mathrm{~nm}$ in the etalon with finesse of 30 . This corresponds to the effective refractive index change of $\Delta \mathrm{n}=3.8 \times 10^{-3}$ and $\Delta \mathrm{n}=1.6 \times 10^{-3}$, respectively. These refractive index changes in $\mathrm{Si}$ is caused by FCC concentrations of $\Delta \mathrm{N}=\Delta \mathrm{P}=1.3 \times 10^{18} \mathrm{~cm}^{-3}$ and $4.8 \times 10^{17} \mathrm{~cm}^{-3}$, respectively. These results show that with greater finesse smaller pump energy is needed.

To better describe the relation between finesse and pump energy, presented in Fig. 9, the experimental investigation and simulations of the influence of pump energy (or power consumption) on the optical modulation of silicon slab resonator with different finesse.

Although the simulations seem to obey the same qualitative functional dependence on finesse as do the 


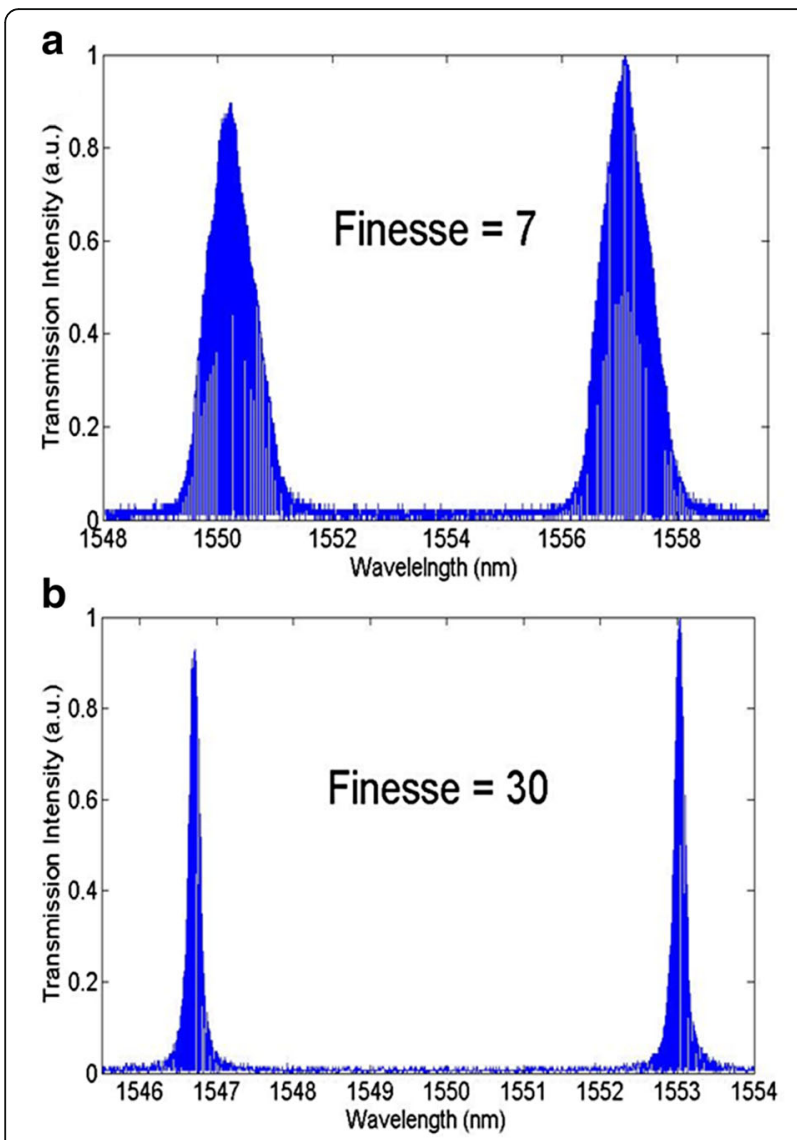

Fig. 8 Transmission intensity vs. wavelength of a coated slab, $50 \mu \mathrm{m}$ thick with finesse of $\mathbf{a} \simeq 7, \mathbf{b} \simeq 30$

experimental results, the energies obtained in experiments are smaller than those from the simulation. These results correspond well with the results from Fig. $5 \mathrm{~b}$ in which the edges of transmission curves of the experiments are sharper than the ones of the simulations.

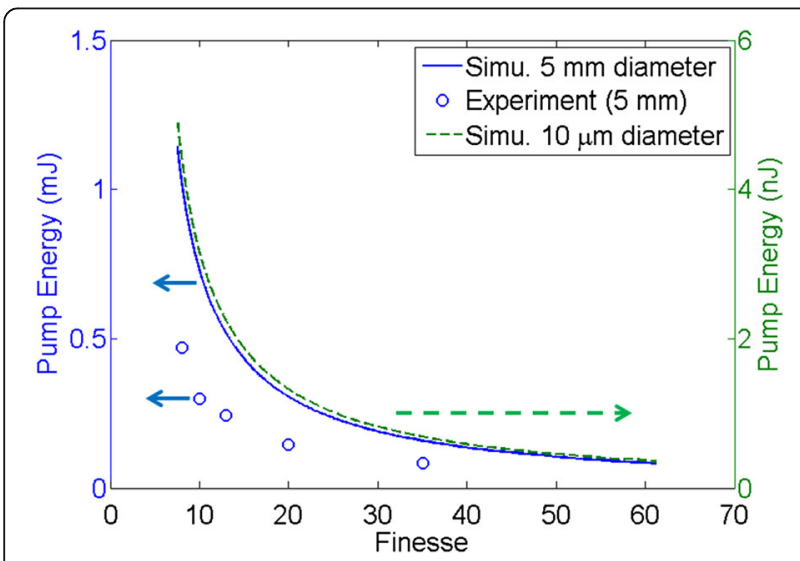

Fig. 9 Pump energy vs. finesse of resonator. Experimental (b/ue circles) and Simulation results for $5 \mathrm{~mm}$ (solid blue line) and $10 \mu \mathrm{m}$ (dashed green line) diameter of pulsed pump beam

\section{Discussion}

The type of challenges and physical phenomena faced in the case of fibre based integration are different from those encountered in the case of waveguides. In the case of fibres the overall power consumption for modulation is significantly larger than in the case of $\mathrm{Si}$ waveguides since the cross section of the optical core is much larger in our case (fibres): waveguide cores are scaled in the hundreds of nanometers whereas fibre cores are scaled in the micron's regime. Therefore, the energy needed for the generation of the same FCC concentration is greater in fibres. This difficulty emphasis the importance of high finesse coated Si slab.

Increasing the finesse, or $\mathrm{Q}$ factor, of our device using coatings means that the modulator can be efficient to be used as a modulator in a fibre system. The advantage of greater $\mathrm{Q}$ factor is that the energy requirements are reduced for the same modulation depth. Another advantage, is that with lower energy consumptions less heat is created, hence, the recovery time decreases, enabling higher operational frequencies and improving the thermal stability.

The FCC life time is mainly determined by the slab thickness, FCC penetration depth and FCC diffusion length. These parameters determine whether the faster surface recombination process or the slower bulk recombination process will be the dominating factor in the FCC life time. In our case, the 10 ns pulse pump permits a $10 \mu \mathrm{m}$ FCC diffusion length into the bulk. Moreover, posterior to the pulse, the FCCs continue their diffusion into the bulk. Hence, after the pulse, the balance between the surface and bulk recombination processes is shifted in favor of the bulk recombination. Thus, for thinner samples the fast surface recombination process will shorten the FCCs life time and consequently will increase the modulation rates.

The light beam of the pump from the input fibre onto the Si slab (see Fig. 2) is about $10 \mu \mathrm{m}$ in diameter. Focusing the pump light beam to $5 \mu \mathrm{m}$ in diameter will reduce the energy consumption for modulation depth of $12 \mathrm{~dB}$ to about $20 \mathrm{pJ}$. In addition, our future research plan includes using thinner Si samples, i.e. few to tens of microns thick, which are characterized with a dominant fast surface recombination over the slow bulk recombination process. The fast surface recombination is in time scale of hundreds of nanoseconds which translates to more than $1 \mathrm{MHz}$ in modulation rate.

On the one hand, because the penetration depth of $532 \mathrm{~nm}$ light beam into the silicon is about $1 \mu \mathrm{m}$, it is preferable that the thickness of the slab will exceed that length so most of the pump energy will be absorbed in the sample rather than passing beyond it. On the other hand, the thinner the sample the faster the recombination process as explained above. From the finesse point of view, even though the power consumption decreases 
with finesse, the rate in which it decreases gets smaller, and becomes insignificant above a finesse of few dozens, as seen in Fig. 9. Therefore, a sample of few microns in thickness with a finesse of about 30 will achieve the best performance of optical modulator in terms of modulation depth, response time and power consumption.

As mentioned in the introduction, the a-Si:H absorption is an order of magnitude larger than that of c-Si. However, when modulating with a resonator, the ER effect has a more dominant influence to the modulation than the EA. For instance, in [13], for modulation depth of $90 \%$ a $\Delta \alpha=$ $23 \mathrm{~cm}^{-1}$ is required, whereas, for a modulation depth of 95\% only a $\Delta \alpha=3 \mathrm{~cm}^{-1}$ because of the additional ER effect in a resonator with finesse of 30 . In addition, due to the poor overlap between the pump light and a-Si:H active region only a fraction of the energy of the pump contribute to modulation. Thus, $1 \mathrm{~nJ} / \mathrm{cm}^{2}$ in [13] or $75 \mathrm{pJ}$ in [12] is needed compared to about $20 \mathrm{pJ}$ for even deeper modulation as calculated above with our device.

In addition to the aforementioned utilities, our silicon slab resonator can also be used as a tunable spectral optical filter realizing narrowband optical transmissions for optics communication [22]. The device we present in this paper can act as a tunable spectral filter with bandwidths lower than $9 \mathrm{GHz}$ FWHM while its tuning can be realized either via the PDE optical modulation mechanism or via the TOE heat modulation.

\section{Conclusions}

In this paper we presented an all-optical modulator based on Si slab resonator for future in-fibre integrated modulations with increased finesse due to coating on both sides of the slab which provides sufficient modulation depth. It was shown that the coating withstands high energy pulse intensities. Moreover, the coating saved us the severe alignment difficulties that were a problem in PDE based conventional etalon modulators. We have shown that the finesse has increased from 2.5 in a bare $\mathrm{Si}$ slab to 30 in the coated one which enabled us to reach modulation depth of $12 \mathrm{~dB}$ on a $\mathrm{Si}$ etalon without the use of waveguides and couplers.

New difficulties in the case of fibre based integration of a Si slab modulator were presented and discussed. PDE based modulation in fibre integrated coated silicon etalon having modulation depth of $12 \mathrm{~dB}$ and full recovery time of 600 ns was shown here for the first time. In this paper we have explained how our device is the first ever efficient PDE based $\mathrm{Si}$ etalon modulator that can be implemented to yield a compact, low power consuming infibre integrated device.

\section{Abbreviations}

CMOS: Complementary metal oxide semiconductor; CW: Continuous wave; EA: Electro-absorption; ER: Electro-refractive; FCC: Free charge carriers;

FP: Fabry-perot; FSR: Free spectral ranges; FWHM: Full width half maximum;
IR: Infra-red; PDE: Plasma dispersion effect; Si: Silicon; SOI: Silicon-on-insulator; TOE: Thermo-optic effect

\section{Funding}

N.A

\section{Availability of data and materials}

Data and materials are available at the lab of Prof. Zalevsky at BIU where the research was performed.

\section{Authors' contributions}

HP and YD performed the numerical and the experimental investigation. HP was leading the writing of the report. MS and MD supervised the experimental investigation. ZZ, MS and MD initiated the idea, the project and supervised the research and the paper writing. All authors read and approved the final manuscript.

\section{Competing interests}

The authors declare that they have no competing interests.

Received: 11 October 2016 Accepted: 16 December 2016 Published online: 19 January 2017

\section{References}

1. van de Lagemaat, J., Park, N.-G., Frank, A.J.: A study by electrical impedance and optical modulation techniques. J. Phys. Chem. B 104(9), 2044-2052 (2000)

2. De Marcellis, A., Palange, E., Janneh, M.: Very high-sensitivity tunable phase detection of light power variations using electrical modulation of Siphotodiode in photovoltaic regime. Electron. Lett. 51(3), 282-284 (2015)

3. Bruck, R., Vynck, K., Lalanne, P., Mills, B., Thomson, D.J., Mashanovich, G.Z., Reed, G.T., Muskens, O.L.: All-optical spatial light modulator for reconfigurable silicon photonic circuits. Optica 3(4), 396 (2016)

4. Hochberg, M., Baehr-Jones, T., Wang, G., Shearn, M., Harvard, K., Luo, J., Chen, B., Shi, Z., Lawson, R., Sullivan, P., Jen, A.K.Y., Dalton, L., Scherer, A.: Terahertz all-optical modulation in a silicon-polymer hybrid system. Nat. Mater. 5(9), 703-709 (2006)

5. Schönenberger, S., Stöferle, T., Moll, N., Mahrt, R.F., Dahlem, M.S., Wahlbrink, T., Bolten, J., Mollenhauer, T., Kurz, H., Offrein, B..: Ultrafast all-optical modulator with femtojoule absorbed switching energy in silicon-on-insulator. Opt. Express 18(21), 22485-22496 (2010)

6. Lee, B.G., Biberman, A., Dong, P., Lipson, M., Bergman, K.: All-optical comb switch for multiwavelength message routing in silicon photonic networks. IEEE Photonics Technol. Lett. 20(10), 767-769 (2008)

7. Liu, A., Rong, H., Paniccia, M., Cohen, O., Hak, D.: Net optical gain in a low loss silicon-on-insulator waveguide by stimulated Raman scattering. Opt. Express 12(18), 4261 (2004)

8. Almeida, V.R., Barrios, C.A., Panepucci, R.R., Lipson, M.: All-optical control of light on a silicon chip. Nature 431(7012), 1081-1084 (2004)

9. Barrios, C.A., de Almeida, V.R., Lipson, M.: Low-power-consumption short-length and high-modulation-depth silicon electrooptic modulator. J. Light. Technol. 21(4), 1089-1098 (2003)

10. Almeida, V.R., Barrios, C.A., Panepucci, R.R., Lipson, M., Foster, M.A. Ouzounov, D.G., Gaeta, A.L.: All-optical switching on a silicon chip. Opt. Lett. 29(24), 2867 (2004)

11. Della Corte, F.G., Rao, S.: Use of amorphous silicon for active photonic devices. IEEE Trans. Electron Devices 60(5), 1495-1505 (2013)

12. Narayanan, K., Elshaari, A.W., Preble, S.F.: Broadband all-optical modulation in hydrogenated-amorphous silicon waveguides. Opt. Express 18(10), 9809 (2010)

13. Rao, S., D'Addio, C., Della Corte, F.G.: All-optical modulation in a CMOS-compatible amorphous silicon-based device. J. Eur. Opt. Soc. Rapid Publ 7(0), 12023 (2012)

14. Gibbs, H.M., Venkatesan, T.N.C., McCall, S.L., Passner, A., Gossard, A.C., Wiegmann, W.: Optical modulation by optical tuning of a cavity. Appl. Phys. Lett. 34(8), 511-514 (1979)

15. Aharoni, R., Baharav, O., Bidani, L., Sinvani, M., Elbaz, D., Zalevsky, Z.: All-optical silicon simplified passive modulation. J. Eur. Opt. Soc. - Rapid Publ. 7, 12029 (2012)

16. Schröder, D.K.: Carrier lifetimes in silicon. IEEE Trans. Electron Devices 44(1), 160-170 (1997)

17. Soref, R. A., Bennett, B. R.: Kramers-Kronig Analysis Of Electro-Optical Switching In Silicon. SPIE Proc. 0704, 32-37 (1987) 
18. Soref, R.A., Larenzo, J.: All-silicon active and passive guided-wave components for $\lambda=1.3$ and $1.6 \mu \mathrm{m}$. IEEE J. Quantum Electron. 22(6), 873-879 (1986)

19. Soref, R.A., Bennett, B.R.: Electrooptical effects in silicon. IEEE J. Quantum Electron. 23(1), 123-129 (1987)

20. Moss, T. S., Ellis, B., Burrell, G. J.: Semiconductor Opto-Electronics. Halsted Press Division, Wiley, London (1973)

21. Cocorullo, G., Rendina, I.: Thermo-optical modulation at $1.5 \mu \mathrm{m}$ in silicon etalon. Electron. Lett. 28(1), 83-85 (1992)

22. Dainesi, P., Kung, A., Chabloz, M., Lagos, A., Fluckiger, P., lonescu, A., Fazan, P., Declerq, M., Renaud, P., Robert, P.: CMOS compatible fully integrated Mach-Zehnder interferometer in SOI technology. IEEE Photonics Technol. Lett. 12(6), 660-662 (2000)

23. Pinhas, H., Bidani, L., Baharav, O., Sinvani, M., Danino, M., Zalevsky, Z: All optical modulator based on silicon resonator. SPIE 9609, 96090L-96090L-7 (2015)

24. Rahman, M.Z.: Modeling minority carrier's recombination lifetime of p-Si solar cell. Int. J. Renew. Energy Res. 2(1), 117-122 (2012)

\section{Submit your manuscript to a SpringerOpen ${ }^{\circ}$ journal and benefit from:}

- Convenient online submission

- Rigorous peer review

- Immediate publication on acceptance

- Open access: articles freely available online

- High visibility within the field

- Retaining the copyright to your article

Submit your next manuscript at $\boldsymbol{s p r i n g e r o p e n . c o m ~}$ 